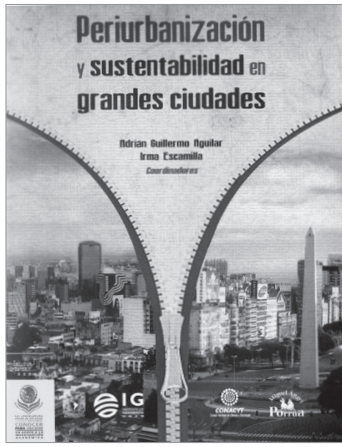

\title{
Periurbanización y SUSTENTABILIDAD EN GRANDES CIUDADES
}

\section{Adrián Guillermo Aguilar e Irma Escamilla (Coordinadores)}

Editor: Universidad Nacional Autónoma de MÉXico (UNAM), Instituto de Geografía

Bajo el título de Periurbanización y sustentabilidad en grandes ciudades, desde México nos llega un libro que entrega importantes aportes teóricos y empíricos sobre la expansión física de las grandes ciudades, la situación que subyace a los procesos de periurbanización, la condición de sustentabilidad y las cuestiones relativas a la gobernanza que en ellas se dan. De algún modo, pese a no ser pionero en el tratamiento de estos temas y aunque está centrado en investigaciones sobre las grandes ciudades mexicanas — salvo la primera parte, donde hay una sección sobre Buenos Aires y otra sobre Santiago de Chile-, se reconoce en este trabajo una aproximación variada en enfoques y de alto valor para comprender los desafíos que en esta materia son hoy comunes en Latinoamérica.
Periurbanización y sustentabilidad... es fruto del IV Seminario Internacional "Procesos metropolitanos y grandes ciudades", organizado por el Instituto de Geografía de la Universidad Nacional Autónoma de México en febrero del año 2009, cuestión que podría significar en muchos casos la compilación de trabajos bajo el formato de Actas de un Congreso. Sin embargo, su estructura y la calidad de la mayoría de los artículos hablan de un acucioso trabajo de selección, calificación y edición por parte de sus coordinadores, Adrián Guillermo Aguilar e Irma Escanilla. Adicionalmente, aun cuando prácticamente todos los investigadores son mexicanos, hecho que podría entenderse como un sesgo en el enfoque de sus trabajos, la pertenencia de cada uno a 
distintos centros de investigación, incluso algunos en calidad de funcionarios públicos, explica en forma importante la amplitud e interrelación de miradas contenidas en esta publicación.

El libro nos ofrece cuatro apartados con quince trabajos. El primer apartado contiene tres artículos sobre urbanización y sustentabilidad en América Latina: uno de los investigadores argentinos Pablo Ciccolella e Iliana Mignaqui, de la Universidad de Buenos Aires; otro de los investigadores chilenos Hugo Romero, Marcela Salgado y Claudio Fuentes, de la Universidad de Chile; y un tercero del investigador Martin Smolka, del Lincoln Institute of Land Policy. Un segundo apartado, con cuatro trabajos sobre metropolización y conflictos ambientales en México, es iniciado por los investigadores Jesús Arroyo e Isabel Corvera, del Departamento de Estudios Regionales de Guadalajara; luego se presenta el trabajo del investigador Ismael Aguilar, del Tecnológico de Monterrey; prosigue con el aporte del investigador Manuel Perló, de la UNAM, y cierra la sección el trabajo de los investigadores Carlos Félix Garrocho Rangel y Juan Campos Alanís, de El Colegio Mexiquense. El tercer apartado, que también contiene cuatro trabajos, se abre con el artículo de dos directores de Oficinas del Distrito Federal, J. Enrique Castelán Crespo y Adolfo Mejía Ponce de León; siguen los investigadores Adrián Guillermo Aguilar y Clemencia Santos Cerquera, de la UNAM; cierran la sección dos trabajos de los investigadores Ma. de Lourdes Rodríguez Gamiño, Jorge López Blanco y Gilberto Vela Correa, el primero de la UNAM y los siguientes de la Universidad Autónoma Metropolitana de Xochimilco. El cuarto y último apartado se inicia con el trabajo de la investigadora Flor M. López de la UNAM, seguido del artículo de la investigadora Ailsa Winston, de la UNAM; el trabajo de los investigadores Antonio Vieyra e Irma Escamilla, de la UNAM, para cerrar con la entrega de los investigadores Rafael Candeau y Sergio Franco, de la Universidad Autónoma del Estado de México de Iztapalapa.

En cuanto a sus contenidos - en términos de aportes teóricos y empíricos-, en cada apartado, y en los trabajos que contienen, se ofrecen interesantes aproximaciones a un conjunto de temáticas que sin duda hoy están presentes no solo en el debate académico, sino también en la agenda pública en Latinoamérica. Es así que Ciccolella y Mignaqui, en el primer apartado, abordan la problemática ambiental de la Cuenca del Río Matanza-Riachuelo en la Región Metropolitana de Buenos Aires desde la perspectiva de la gobernanza, mostrando la fractura institucional, la superposición de jurisdicciones y la errática conducción de la política urbana que condena actualmente a una población de casi medio millón de habitantes de catorce municipios a vivir en condiciones de alta vulnerabilidad social y deterioro ambiental. Sobre la base de un análisis del marco jurídico-institucional, los autores no se quedan solo en el diagnóstico, sino que lo ven como una oportunidad y apuntan a la necesidad urgente de una agenda ambiental metropolitana como punto de partida de la solución.

Puesto el foco al otro lado de la cordillera de los Andes, el trabajo de Romero, Salgado y Fuentes analiza el problema de la segregación socioambiental en Santiago de Chile, para lo que se sirve de un estudio de caso en la comuna de Peñalolén, utilizando un sistema de información geográfica y combinando el análisis ambiental con el social. Los resultados de esta investigación dan cuenta 
de que las nuevas formas de segregación no solo se asocian a exclusiones del mercado residencial, laboral, educacional o cultural, sino que son de orden ambiental, y tocan aspectos como los niveles de contaminación y la localización en zonas de riesgo, entre otros. Se cierra este apartado con el trabajo de Smolka, el cual, basado en diversos antecedentes de distintas ciudades de América Latina provistos por otros investigadores, examina cómo la falta de suelo urbano y el alza en sus precios responde en gran parte a la negligencia de los gobiernos en cuanto a la utilización de las tierras públicas y a cierta parcialidad en el ejercicio de la regulación urbanística.

En el segundo apartado, Arroyo y Corvera comparan la gobernanza ambiental en tres importantes zonas metropolitanas de México: la de Guadalajara (ZMG), la de Monterrey (ZMM) y la de Puebla-Tlaxcala (ZMPT). Las variables que utilizan para medir tal condición son las tasas de crecimiento de población y de superficie, la localización de plantas de tratamiento de residuos sólidos, los reglamentos, la participación ciudadana, así como datos en materia de mejoramientos y problemáticas ambientales. Los resultados muestran contrastes importantes; por ejemplo, que la ZMG presenta un mayor deterioro ambiental en comparación con la ZMM y la ZMPT, aunque la primera demuestre mayor capacidad para promover la participación ciudadana. Concluyen afirmando la necesidad de que los gobiernos locales aborden estas problemáticas en conjunto, a escala metropolitana y regional.

A continuación, Aguilar Barajas propone un análisis hemerográfico sobre el proceso de metropolización de Monterrey, específicamente sobre el caso del Arco Vial Sureste.
El aporte principal de este trabajo está en que muestra cómo proyectos de este tipo muchas veces se encuentran lejos de una planeación y evaluación integrada en el territorio metropolitano, donde el propósito de la conectividad se prioriza por sobre lo ambiental, lo que además genera problemas de gobernabilidad entre las distintas escalas de gobierno y conflictos con la ciudadanía.

Un tercer trabajo de este apartado, de Perló, explora una temática de vital importancia para la Ciudad de México, como es la urgente necesidad de recuperar la sustentabilidad hídrica. A partir de un concepto que apuesta a un aprovechamiento del recurso agua en forma más eficiente y equitativa, el autor revisa los esfuerzos gubernamentales en esta materia desde el año 2007, avanza en algunas propuestas específicas que se inscriben en lineamientos de políticas, concluyendo que es posible la recuperación de la sustentabilidad hídrica para esta gran metrópolis.

Se cierra este segundo apartado con un trabajo de Garrocho y Campos que escapa a las temáticas propiamente ambientales, ya que se focaliza en estudiar la estrategia espacial de las cadenas de farmacias en el área metropolitana de Toluca. Con base en el enfoque de economías de aglomeración, definen los factores clave que guían a las tres principales cadenas de farmacias y visualizan los patrones espaciales de localización, concluyendo en que hay una suerte de imitación locacional entre ellas.

El tercer apartado del libro se inicia con el trabajo de Castelán y Mejía Ponce sobre la política ambiental en el suelo de conservación en el Distrito Federal. El aporte de este trabajo lo constituye la revisión e identificación de factores coadyuvantes del cambio de usos de suelo para explicar el origen de la 
amenaza sobre estos suelos, así como destacar el Plan Verde 2007-2022 para avanzar hacia la sustentabilidad de su desarrollo y de sus programas hoy en curso. El estudio concluye planteando que el reto de la sustentabilidad requiere sumar actores al esfuerzo del Gobierno del Distrito Federal para garantizar su futuro.

Siguen Aguilar y Santos con una presentación sobre el manejo de reasentamientos humanos irregulares en el suelo de conservación del Distrito Federal, en la cual se analiza la efectividad de la política urbana. Para tal propósito, revisan exhaustivamente el estado actual de la temática en el mundo y, específicamente, el rol de los programas existentes en materia de política urbana desde mediados de los años noventa en México. A la luz de los antecedentes que plantean, concluyen que a pesar del aumento de asentamientos humanos irregulares, tanto el Distrito Federal como las Delegaciones carecen de una política urbana eficaz para enfrentar este desafío.

Un tercer trabajo, de Rodríguez, López y Vela, sobre suelos de conservación en el Distrito Federal, pone el énfasis en el crecimiento urbano y deterioro ambiental, revisando cartográfica y estadísticamente los cambios producidos en la cobertura vegetal y el uso de suelo. Los mismos autores cierran este apartado con un aporte sobre calidad de suelos para el aprovechamiento sustentable de la Sierra de Guadalupe en el Distrito Federal, realizado mediante un trabajo de campo y apoyo de laboratorio. Especial énfasis ponen en el análisis de los suelos deforestados, mostrando que los programas de forestación que los preceden han privilegiado especies que se adaptan menos naturalmente a las condiciones edáficas de la sierra.
La última parte del libro se inicia con un trabajo de López sobre el agua y las condiciones de salud en la periferia urbana pobre del Distrito Federal, donde se releva la importancia de la perspectiva geográfica en este tipo de estudios. Junto con entregar un panorama sobre las condiciones de vida en la periferia urbana del Distrito Federal, especialmente para el caso de la población que vive en asentamientos irregulares, se muestra una importante cartografía sobre distintas variables de salud que tienen un lugar en las desigualdades existentes. Entre las principales conclusiones que entrega este estudio está la constatación de que el débil panorama socioterritorial, unido a una mala calidad de los servicios prestados, son factores que inciden en el deterioro de la salud de la población de la periferia urbana.

En el artículo siguiente, Winston estudia la problemática relativa a la reubicación de asentamientos irregulares en la Delegación de Tlalpan, en el Distrito Federal, derivada de la expansión urbana periférica de la Ciudad de México. Un acento importante de su investigación está en el reconocimiento de aspectos relacionados con la comunidad, el vínculo entre redes sociales y el tema de inseguridad que subyacen a los asentamientos irregulares. Para el caso de Tlalpan, se aprecia que el programa de reubicación ha permitido una recuperación del suelo de conservación; sin embargo, la autora plantea que los fines ambientales deben ir de la mano de la consecución de fines sociales para un desarrollo sustentable.

Un tercer trabajo, de Vieyra y Escamilla, analiza el efecto de los proyectos productivos y sus implicancias en el proceso de urbanización en Milpa Alta en el Distrito Federal. Junto con analizar las tendencias en las tasas de crecimiento de la población 
entre 1990 y 2005 , se revisa para el mismo período los cambios en la población económicamente activa, la proliferación de asentamientos irregulares y los proyectos de inversión en diferentes sectores productivos por delegación. Una de las conclusiones más importantes de este trabajo radica en reconocer que iniciativas tendientes a generar proyectos productivos pueden ayudar a mitigar los impactos de la expansión urbana en suelos de conservación, particularmente vinculados a la dinámica del desarrollo rural.

Para finalizar, este cuarto apartado se cierra con el trabajo de Candeau y Franco, quienes, también en la problemática de los asentamientos humanos irregulares en Milpa Alta, plantean el análisis de los geosistemas urbanos. Desde un enfoque geográfico, buscan comprender las consecuencias e impactos de los problemas medioambientales en la sociedad; para ello examinan un conjunto de variables por tema, levantadas en terreno, que comparan la situación entre distintos asentamientos humanos irregulares. En conclusión, esta investigación aporta importantes similitudes y contrastes entre los geosistemas urbanos observados.

En resumen, a partir de la revisión de los trabajos presentados en este libro, se reitera que son un aporte sustantivo a la literatura sobre las problemáticas ambientales y su relación con la expansión urbana, la periurbanización y la gobernanza en las áreas metropolitanas, especialmente de México. Se destaca especialmente el apartado tres, donde los trabajos se interrelacionan de mejor manera que en los otros apartados, sin restarles mérito a otros trabajos que son parte integral de este libro. CEURE

\section{Arturo Orellana}

Pontificia Universidad Católica de Chile.

Santiago, Chile

E-MAIL:AMORELLA@UC.CL 